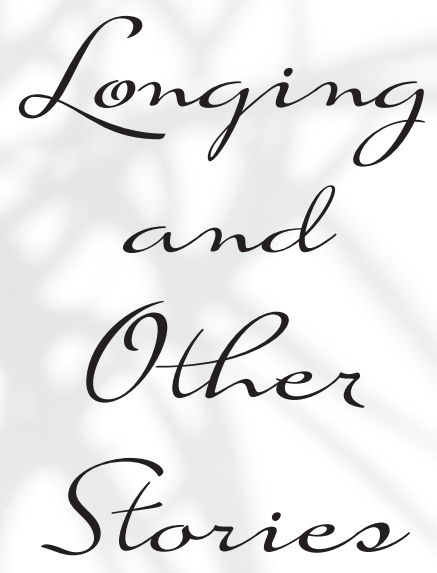

Weatherhead Books on Asia

Weatherhead East Asian Institute, Columbia University 


\section{Weatherhead Books on Asia \\ Weatherhead East Asian Institute, Columbia University}

For a list of titles in the series, see page 149. 


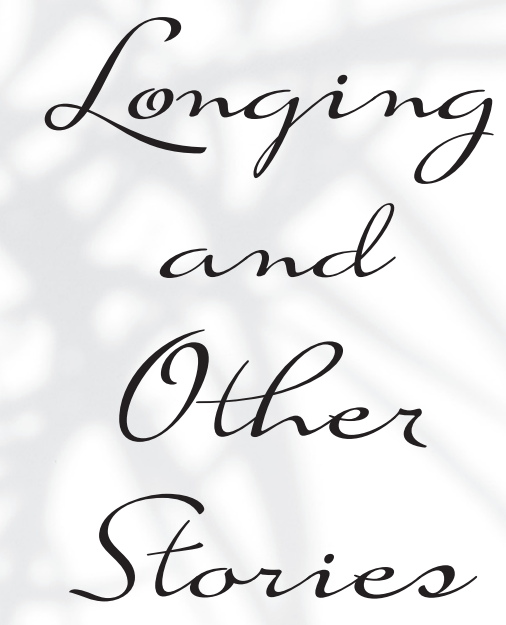

\title{
Jun’ichirō Tanizaki
}

\author{
Translated by \\ Anthony H. Chambers \& Paul McCarthy
}

Columbialmiversitypess New Pork 
This publication has been supported by the Richard W. Weatherhead Publication Fund of the Weatherhead East Asian Institute, Columbia University.

Columbia University Press wishes to express its appreciation for assistance given by the Pushkin Fund in the publication of this book.

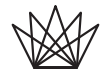 \\ Columbia University Press \\ Publishers Since 1893 \\ New York Chichester, West Sussex \\ cup.columbia.edu \\ Copyright (C) 2022 Columbia University Press \\ All rights reserved
}

Library of Congress Cataloging-in-Publication Data

Names: Tanizaki, Jun'ichirō, I886-I965, author. | Chambers, Anthony H.

(Anthony Hood), translator. McCarthy, Paul, I944- translator.

Title: Longing and other stories / Jun'ichirō Tanizaki ; translated by

Anthony H. Chambers and Paul McCarthy.

Description: New York : Columbia University Press, [2022]

Identifiers: LCCN 20210I8905 (print) | LCCN 2021018906 (ebook) |

ISBN 9780231202145 (hardback) | ISBN 9780231202152 (paperback) |

ISBN 97802315544II (ebook)

Subjects: LCGFT: Short stories.

Classification: LCC PL839.A7 L66 2022 (print) | LCC PL839.A7

(ebook) | DDC 895.63/44-dc23

LC record available at https://lccn.loc.gov/2021018905

LC ebook record available at https://lccn.loc.gov/2021018906

Columbia University Press books are printed on permanent and

durable acid-free paper.

Printed in the United States of America

Cover design: Lisa Hamm

Cover image: Evening Cool, I925, Ito Shinsui (1898-I972),

Arthur M. Sackler Gallery, Smithsonian Institution,

Robert O. Muller Collection, Bridgeman Images. 